\title{
New forms of induction of apoptosis in aggressive lymphoma using peptides that interrupt the RAS / RAF interaction
}

\author{
C Bergna ${ }^{1}$, G H Marin ${ }^{1,2}$, M Maiz $^{1}$, H Bruzzoni-Giovanelli ${ }^{3}$, C Ponzinibbio ${ }^{1}$, G Schinella ${ }^{1,4}$, J Errecalde $^{1}$, \\ A Rebollo ${ }^{5}$
}

(Index words: T-LYMPHOMA, NHL-T, Peptides, Apoptosis)

\begin{abstract}
RAS-RAF-MEK-ERK is a key pathway for apoptosis regulation in cancer cells. B-Raf-inhibitors such as PLX4032 peptide was developed by Institute CurieUniversité Pierre et Marie Curie in order to induce apoptosis in cancer cells.

Objective: To demonstrate pro-apoptotic properties and survival outcome of EP2014/064243 peptide in murine aggressive lymphoma.

Material and methods: BALBc mice with T-lymphoma were randomized assigned either in Group $A$ (peptide+cyclophosphamide-CFM); Group B (peptides), Group C (CFM-control) or Control D (Cl-Na 0.9\%-SF control group). Survival probability was calculated by Kaplan-Meier analysis. Apoptosis was detected using TUNEL technique. The protocol was approved by the Institutional Committee for Animal Care (CICUAL: T04-01-2015)

Results: The median survival was 24 days (21.6-26.4) for placebo, 33 days (28.0-35.4) for the CFM monotherapy group, 33 (27.1-35.8) for the peptide group and 34 days $(24,4-40)$ for CFM-peptide combined treatment $(p<0.05)$. In lymph node tissue the mean TUNEL positive cells per field for each treatment group was 2, 12 and 13 and 35 for SF, CFM, peptide and combined therapy $(p<0.05)$.

Conclusion: These findings suggest that in murine aggressive lymphoma treated by an experimental peptide in addition with CFM, had an exponentially pro-apoptotic effect than CFM alone, suggesting that the peptide potentiated the anti-tumoural effect of CFM.
\end{abstract}

Ceylon Medical Journal 2019; 64: 45-51

DOI: http://doi.org/10.4038/cmj.v64i2.8890

\section{Introduction}

Murine haematological malignancies models can be homologated to the human ones due to the similarities between the two species. These alternative models constitute a valuable tool to explore pathophysiology of different neoplasias in order to assay new therapeutic modalities. In this regard, a murine BALBc T-lymphoma with aggressive clinical behaviour, provided by the Pathology Department of the National University of La Plata, Argentina, became an ideal experimental model that correlates to human aggresive lymphoid leukemias.

One of the pathways to study by this model is the Ras signal transduction for apoptosis regulation. Ras is a GTPase family located at the inner leaflet of the plasma membrane, Ras binds to one of their multiple effectors, Raf serine/threonine kinase and induces their translocation to the plasma membrane where Ras is fully activated $[1,2]$. RAS/RAF/MEK/ERK is the signal transduction pathway in diverse types of cells including malignant lines.

RAS-RAF-MEK-ERK pathway has been considered a promising target for anticancer therapy [2]. B-Rafinhibitors such as PLX4032 molecules are under investigation in clinical trials. In this sense, the Institute Curie- Université Pierre et Marie Curie (Paris 6) in France, mapped binding sites of K-Ras to B-Raf and designed peptides in order to disturb Ras/Raf interaction which showed pro-apoptotic properties. These peptides were patented as WO2015001045 A2 (PCT/EP2014/064243) [3,4]. The invention thus provides a chimeric peptide capable for cell penetration and with pro-apoptotic properties.

In order to demonstrate the activity of these peptides against aggressive murine lymphoma, we performed the present study comparing the overall survival of mice

\footnotetext{
${ }^{1}$ Faculty of Medical Science, National University of La Plata, ${ }^{2}$ CONICET-UNLP, ${ }^{3}$ Université Paris 7, Hôpital Saint Louis, Pharmacology, Centre d'Investigations Cliniques, ${ }^{4} \mathrm{CIC}$, Scientific Research Commission, Buenos Aires, ${ }^{5} \mathrm{CIMI}$, Inserm/UPMC/CNRS, Université Pierre et Marie Curie, Paris 6, France; Centre d'Immunologie et des Maladies Infectieuses.

Correspondence: GHM, e-mail: <gmarin2009@gmail.com>. Received 11 September 2018, revised version received 19 December 2018 and accepted 11 March 2019.
}

This is an open-access article distributed under the terms of the Creative Commons Attribution License, which permits unrestricted use, distribution, and reproduction in any medium, provided the original author and source are credited. 
affected by lymphoma receiving either a classical treatment (based on cyclophosphamide infusion); placebo; or peptide EP2014/064243 that induce apoptosis and cell cycle arrest.

\section{Method}

Murine lymphoma model: From the finding of a spontaneous lymphoma in a male adult mouse of endogamic Balb/c strain grew up under free specific pathogens standard conditions, neoplastic cells were obtained from inguinal, axillaries and cervical lymph nodes. The model was maintained by successive passages to inbred male mice of the same strain, maintained under conditions of sterility (bed, water and food) until the development of visible and palpable adenomegalies was detected. The protocol was approved by the Institutional Committee for the Care of Laboratory Animals of the Faculty of Medicine of the UNLP (Protocol No. T04-012015).

Tumour infusion: To obtain the neoplastic tissue, euthanasia was performed by cervical dislocation and extraction of inguinal, axillary and cervical lymph nodes with aseptic technique. They were processed by mechanical attrition and filtration through $90 \mu \mathrm{m}$ metal mesh and washed with PBS by centrifugation for $10 \mathrm{~min}$ at $2000 \mathrm{rpm}$ until obtaining cell suspension of lymphomaderived cells. A suspension containing $3 \times 10^{6}$ nucleated cells obtained from in healthy, male, were inoculated intraperitoneally to 45 day Balb / c mice which were maintained under conditions of sterility (bed, water and food) until development of visible adenomegalies. Twentyfour male mice specimens were infused with lymphoma cells using the described process.

Groups of study: The 24 mice with clinical evidence of lymphoma (palpable lymph nodes) at 14 days after inoculation of neoplastic cells, were randomized into four groups of 6 mice each (Table 1). Group A received the combination of intravenous (iv) peptide at $6 \mathrm{mg} / \mathrm{kg} /$ weekly plus intraperitoneal (ip) cyclophosphamide (CFM) (Filaxis ${ }^{\circledR}$ ) at $50 \mathrm{mg} / \mathrm{kg}$ per week. Group B received peptide at $6 \mathrm{mg} / \mathrm{kg} /$ weekly (iv) per week. Group C received cyclophosphamide (CFM) (Filaxis ${ }^{\circledR}$ ) at $50 \mathrm{mg} / \mathrm{kg}$ ip per week. Group D received Cl-Na 0.9\% placebo solution (ip and iv) at weekly frequency. (Groups C and D were considered controls). The peptide was designed and provided by Angelita Rebollo (from Marie Curie Institute, Paris-France). This frequency of drug administration was chosen from preliminary experiments. The optimal dose of CFM was obtained from previous trials.

Pharmacological induction of apoptosis: an experimental peptide developed by chemical synthesis at the Institute Curie- Université Pierre et Marie Curie (Paris 6), France, patented as EP2014/064243 was tested in order to demonstrate the ability of the Ras/Raf interaction with pro-apoptotic properties (Groups A and B).
Variables of study: The trial was carried out to determine variables like overall survival in treated animals or level of apoptosis induced.

Survival analysis: The animals were kept in sterile conditions (bed, water and food) and left to natural evolution to compare survival. The overall survival probability was calculated by Kaplan Meier analysis comparing the medians among the different groups.

Table 1. Batch distribution of treatment groups

\begin{tabular}{lll}
\hline Group & Day 1 & Day 2 \\
\hline $\begin{array}{l}\text { Group A: Treated } \\
\text { CFM+peptide }\end{array}$ & CFM 50mg/kg ip & peptide $6 \mathrm{mg} / \mathrm{kg}$ iv \\
$\begin{array}{l}\text { Group B: Treated } \\
\text { peptide }\end{array}$ & SF ip & peptide $6 \mathrm{mg} / \mathrm{kg}$ iv \\
$\begin{array}{l}\text { Group C: Control } \\
\text { CFM }\end{array}$ & CFM 50mg/kg ip & SF iv \\
$\begin{array}{l}\text { Group D: Control } \\
\text { Placebo (SF)Cl-Na }\end{array}$ & SF ip & \\
\hline $\begin{array}{l}\text { Treatment cycles were repeated weekly. CFM: cyclophosphamide; } \\
\text { SF (0.9\% Cl-Na Solution); ip (intraperitoneal administration) }\end{array}$
\end{tabular}

Apoptosis quantifications: in order to determine the kinetics of pro-apoptotic action, another experiment was carried out in which the 4 random groups of treatment were segregated after 14 days of inoculation with the lymphoma. 48 hours after the treatment cycle was finished, the animals were sacrificed by cervical dislocation and lymph node and spleen samples were obtained and included in paraffin for histological processing and detection of apoptosis demonstrated by TUNEL quantification.

\section{Results}

\section{Clinical features this type of " $t$ " lymphoma}

After intraperitoneal inoculation of $3 \times 10^{6}$ nucleated cells in healthy adult Balb/c males, $100 \%$ of the animals developed lymphoma. The time of evolution until the development of clinical manifestations is variable according to the number of tumour cells inoculated. For the amount of cells inoculated in this work, an average of 8.4 days passed until tumour detection. Initially, it was observed evidence of enlargement of the superficial lymph nodes (cervical, axillaries and inguinal nodes) becoming perceptible to the external palpation, less frequently it was observed abdominal lump that denotes the presence of splenomegaly. The appearance of anaemia, detected through ocular pallow, and bristling of hair continues to be visible. In advanced phases, alterations of behaviour, posture and neurological signs like ataxia or paralysis and respiratory difficulty appear.

The basal median overall survival was measured in study from a control group of untreated animals that developed the neoplasia $(n=8)$ using the Kaplan Meier method. The average result was 23 days (19-33) (Figure 1). 
Overall Survival Kaplan-Meier

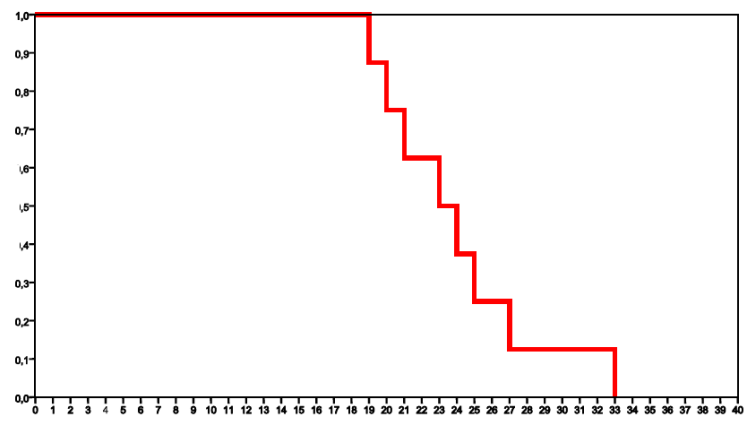

Days from inoculation

Figure 1. Probability of survival of mice that develop lymphoma. Model Kaplan-Meier. $\mathbf{N}=8$.

Necropsy revealed generalized adenomegalies with hard-elastic consistency, and whitish colour, some of them with necrohemorrhagic changes. Splenic involvement was consistently seen. Other organs (liver, kidneys, thymus) showed diffuse enlargement.

\section{Histological description}

Histopathological analysis with hematoxylin-eosin revealed distortion of the ganglionic architecture by diffuse lymphoid infiltration formed by a monomorphic cell population consisting of medium-sized cells, with little agranular basophil cytoplasm, lax chromatin indented nuclei, and central lymphoblastic nucleoli (Figure 2). According to the cytomorphological analysis performed on the ganglion imprint, the average cell diameter was $8.96 \mu \mathrm{m}$ (range 5.8-16.4 $\mu \mathrm{m}$ ) and the average cell area was $76.74 \mu \mathrm{m} 2$ (range 30.37-244.95 $\mu \mathrm{m} 2$ ). 25 mitotic figures were shown per $400 \times$ large field and the presence of macrophages with tinged bodies interspersed between the lymphoid fundus (Figure 3 and Figure 4).

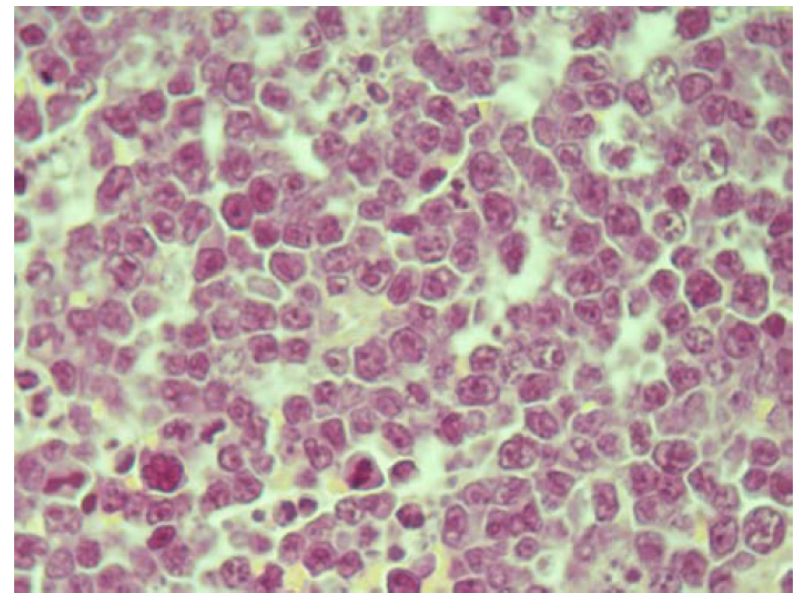

Figure 2. Murine lymphoma H-E 1000x ganglionic histology. Diffuse infiltration by medium size lymphocytes with lax chromatin nuclei with central lymphoblastic nucleoli. Some mitotic figures and macrophages with tingible bodies are visualized.

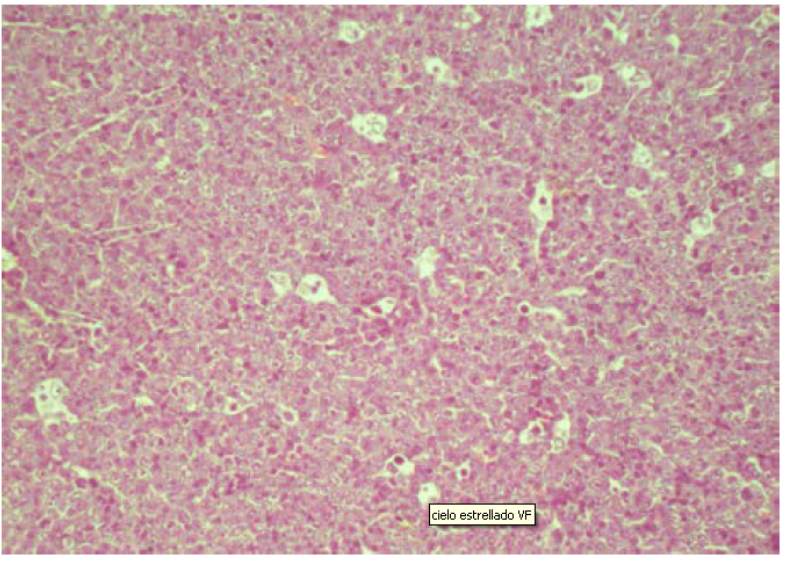

Figure 3. Murine lymphoma H-E 100x nodal histology. Diffuse lymph node infiltration due to high grade lymphoma.

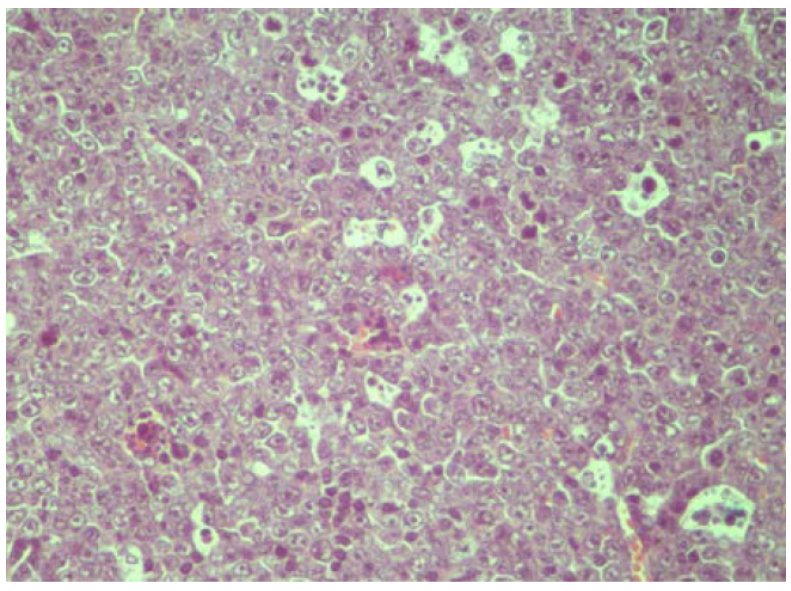

Figure 4. Murine Iymphoma H-E 400x nodal histology. Pattern in starry sky. Infiltration diffuse lymphoblastic-like cells are visualized mitotic figures and macrophages with tangible bodies.

The involvement of bone marrow and spleen was diffuse, and predominance of white pulp in the latter. The thymus is also compromised, showing predominantly perivascular neoplastic cell nodes, as well as nonlymphoid organs (liver, kidney, brain). In peripheral blood of all mice it was detected atypical circulating lymphoma lymphocytes.

\section{Inmunophenotype}

The immunophenotypic characterization of the lymphoma revealed to be of lymphoid origin $\mathrm{T}$ since the expression of CD3 was intense and diffuse throughout the lymph node structure (Figure 5), whereas expression of CD45R / B220, pan B marker, was weak and limited to peripherals patches (Figure 6). 


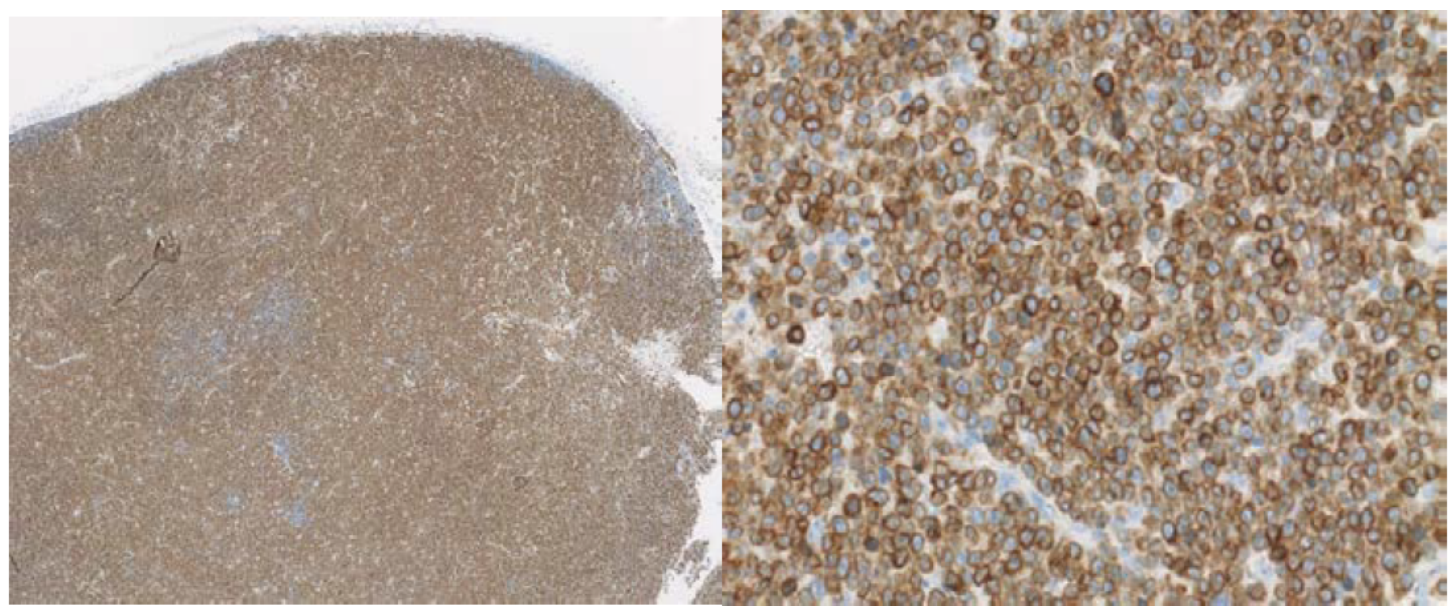

Figure 5. Lymph node immunophenotype. IHQ Anti-CD3 Intense diffuse lymph node positivity. Left: 200x. Right: 400x.

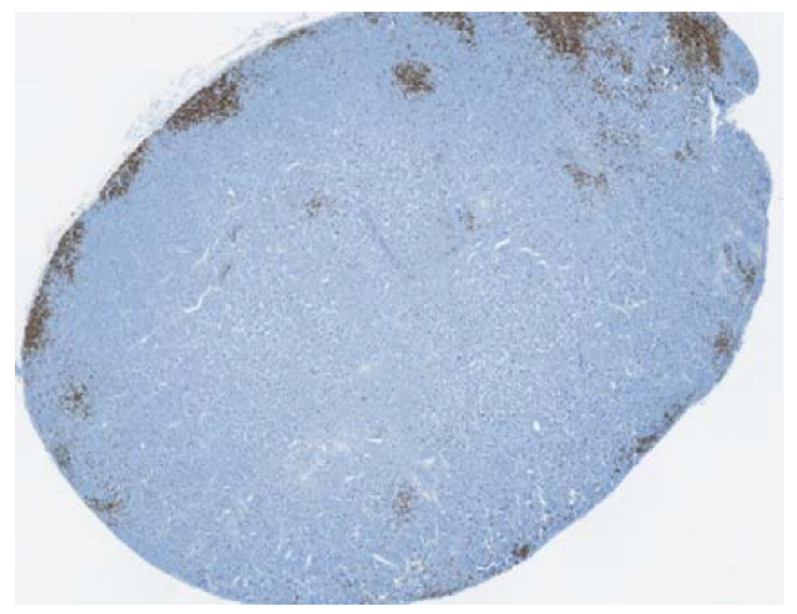

Figure 6. Immunophenotype Iymph node. IHQ Anti-CD45R / B220. Negative, weak presence patched in the periphery of the tissue. 200x.

\section{Pharmacological results}

\section{A) Survival analysis}

For the evaluation of survival, the animals of the different treatment groups received weekly cycles from day 14 until dead. For all batches, the average treatment cycles received was 3 , with a range of 1 to 4 cycles. The lower limit was conditioned by survival and the upper limit of 4 cycles was arbitrarily defined by availability of the experimental peptide.

In Figure 7, is shown the distribution of the overall survival for the different groups using the Kaplan Meier model. Comparatively, the median survival was 24 days (21.6-26.4) for the SF controls, 33 days (28.0-35.4) for the CFM monotherapy group, 33 (28.1-35.8) for the peptide group and 34 days $(24,4-40)$ for animals with combined treatment with CFM and inhibitor peptide ( $\mathrm{p}<0.05)$. It should be noted that no animal lived more than 40 days.
Overall Survival Kaplan Meier

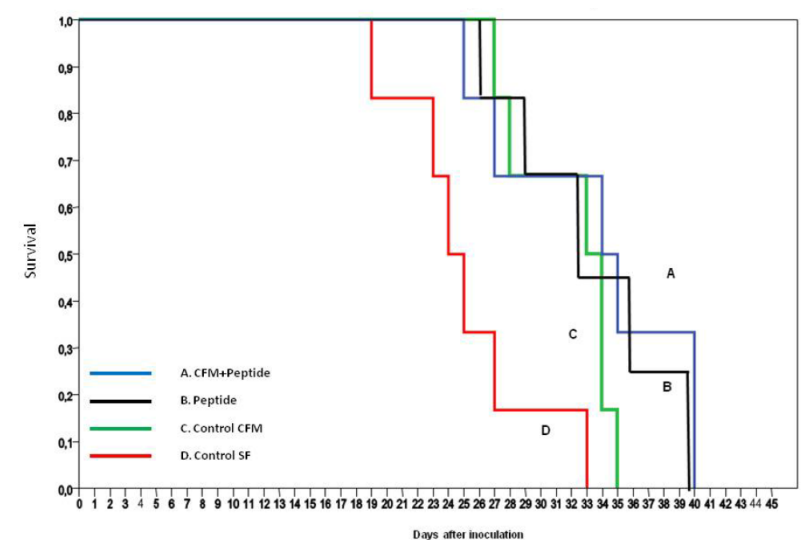

Figure 7. Probability of survival. Kaplan-Meier Model $\mathbf{N}=6$.

Comparative survival

\begin{tabular}{|c|c|c|c|}
\hline & Square Chi & $\mathrm{gl}$ & Sig \\
\hline Log Rank (Mantel-Cox) & 11,246 & 2 & .004 \\
\hline $\begin{array}{c}\text { Broslow } \\
\text { (Generalized Wilcoxon) }\end{array}$ & 10,495 & 2 & .005 \\
\hline Tarone-Ware & 10,828 & 2 & .004 \\
\hline
\end{tabular}

\section{B) Quantification of apoptosis}

In the second stage, the number of cells in apoptosis (TUNEL positive) per field of high magnification $(400 \times)$ in lymph node and spleen samples was performed 48 hours after the end of the treatment.

In lymph node tissue the mean TUNEL positive cells per field for each treatment group was 2 for the SF treated group, 12 and 13 for the groups treated with CFM and experimental peptide in monotherapy respectively, and 35 
for the group receiving treatment combined (Figure 8 and Figure 9). Comparatively the 3 groups of treated animals had significantly higher apoptosis compared to controls receiving $S F(p<0.05$ ). In addition, the group treated with the combination of CFM and experimental peptide was significantly higher in number of apoptotic cells per field than mice receiving the active agents CFM in monotherapy ( $\mathrm{p}<0.05$ ). No difference was observed comparing the animals treated with CFM+peptide group vs peptide inhibitor monotherapy alone.

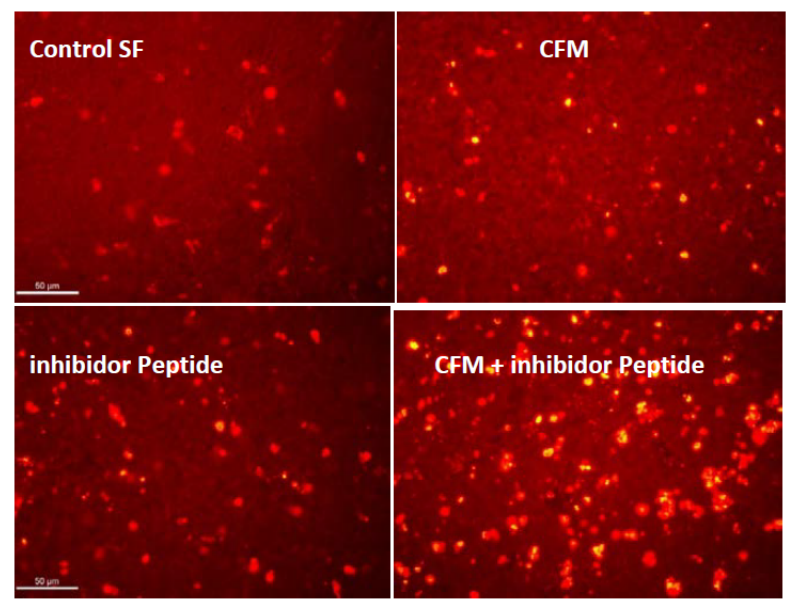

Figure 8. TUNEL in lymph node at 48 hours of 1 cycle of treatment. Apoptotic (yellow) cells are observed per large increase field in the different treatment groups. 400x.

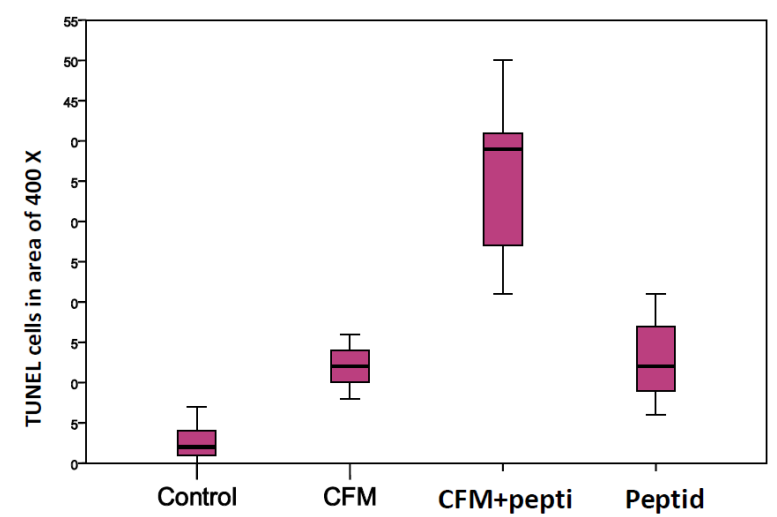

Figure 9. TUNEL Iymph node at $\mathbf{4 8}$ hours of 1 cycle of treatment. Distribution of the number of TUNEL positive cells per large increase field in the different treatment groups is shown.

In splenic tissue the mean number of TUNEL-positive cells per high-magnification field was 13 for controls with SF, 17, 11 for those receiving inhibitor peptide and CFM monotherapy respectively and 19 for those receiving combination therapy (alkylating agent and the experimental peptide) (Figure 10). The comparative analysis between means of the different groups was not statistically significant

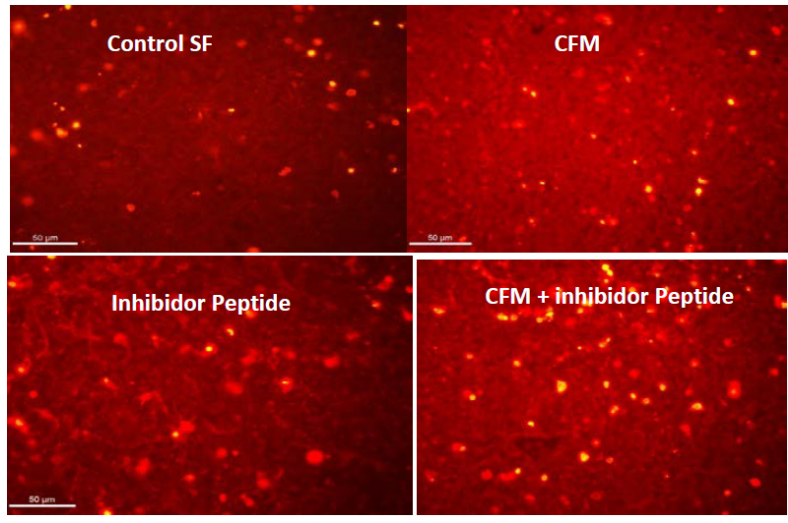

Figure 10. TUNEL in splenic tissue at 48 hours of 1 treatment cycle. Apoptotic (yellow) cells are observed per large increase field in the different treatment groups. $400 \times$.

\section{Discussion}

In vitro results with inhibitor peptide showed that this could be a feasible strategy for the treatment of cancer by sensitizing tumour cells to the process of apoptosis [5-10]. In a previous work our group used this experimental peptide in monotherapy in another model of murine leukaemia/lymphoma $\mathrm{T}$, inoculated daily ip by doses of $2 \mathrm{mg} / \mathrm{kg}$ [11]. This work found that the peptide was able to prolong survival in relation to control mice inoculated with saline. In our trial, it was found convenient to apply it in an ip form given the peptidic nature of the compound. In animals with lymphoma after an experimental peptide dose, there was an increase in the number of apoptotic cells compared to SF controls, in lymph node tissue obtained $48 \mathrm{~h}$ after inoculation.

In addition, inhibition of apoptosis was shown to increase cell sensitivity to antineoplastic drugs and has the advantage of acting through mechanisms other than conventional cytotoxic drugs. CFM is an alkylating prodrug that causes DNA damage leading to cell death by apoptosis. DNA damage sensed by p53 stops the cell cycle at the G1 / S checkpoint. At this point, the cell can repair the alkylation or suffer death by apoptosis. The lethality of DNA alkylation depends on the recognition, the formation of breaks in the DNA chain by repairing enzymes and an intact apoptotic response. Thus, it might be hypothesized that the action of the alkylating agent could be potentiated by generating a proapoptotic state with the use of the experimental peptide that inhibits apoptosis. The mechanisms of cell death triggered by CFM are not known in detail, however, studies in cell lines showed that activation of apoptotic is triggered by mitochondrial pathway that dependent of caspase activation.

In this preclinical trial, combined treatment with CFM and apoptosis inhibitor peptide induced increased lymph node apoptosis 48 hours after the end of treatment and 
significantly prolonged overall survival with respect to control animals. In addition, the proapoptotic effect at the ganglion level was significantly higher compared to their respective monotherapies. This finding suggests that both treatments may be synergistic.

Noteworthy, the same analysis in splenic tissue showed no difference between the treated groups compared to controls. This could be due to the heterogeneity of the lymphoid infiltration by the lymphoma, with dominant involvement of the white pulp and the persistence of large numbers of cells of the monocytemacrophage system in the splenic tissue.

\section{Conclusions}

We present an original model of murine $\mathrm{T}$ cell lymphoma, satisfactorily established in male offspring of the Balb/c strain. It presents aggressive clinical behaviour with a short survival, high mitotic index, a pattern in starry sky and manifesting with generalized adenomegaly, splenomegaly and variable neurological compromise. It presented with a diffuse involvement of lymphoid organs and focal perivascular involvement of the thymus and non-lymphoid organs, with an unstable presence of atypical cells in the peripheral blood.

This lymphoma was used in this study as a preclinical model described by assaying a pro-apoptotic experimental peptide inhibitor in association with CFM in order to produce a sensitizing effect. Combined treatment in weekly cycles resulted in longer survival compared to controls treated with either alkylating agent or placebo. The quantification of apoptosis in the lymph node 48 hours after the end of the treatment cycle was significantly superior for the groups that received inhibitor peptide with or without CFM, compared to control groups. In addition, in this tissue, the combination of experimental peptide and CFM produced higher apoptotic cells per field compared to the respective monotherapies.

These findings suggest that in murine lymphoma cells treated by an experimental peptide in addition with CFM, had greater pro-apoptotic effect than CFM alone, suggesting that both treatments are synergistic. This finding opens new insights in cancer therapeutic tools.

\section{Acknowledgment}

The authors are grateful for the support of the Sorbonne Paris Cité University Group and CIN for the development of this research.

\section{References}

1. Maurer G, Tarkowski B and Baccarini M. Raf kinases in cancer-roles and therapeutic opportunities. Oncogene 2011; 30: 3477-88.

2. Baines AT, Xu D, Der CJ. Inhibition of Ras for cancer treatment: the search continues. Future Med Chem 2011; 3(14): 1787-808.

3. Rebollo A, Nemati F, Decaudin D. Pro-apoptotic ras and raf peptides WO 2015001045 A2. Universite Pierre Et Marie Curie (Paris 6), Institut Curie, 2016.

4. Tian L, Zhang X, Nemati F, et al. Identification of ras/raf binding site and design of interfering peptide with potential clinical application. Integr Mol Med 2016; 3(6): 1-9.

5. Hung MH, Chen KF. Reprogramming the oncogenic response: SET protein as a potential therapeutic target in cancer. Expert Opin Ther Targets 2017; 21, 7: 685-94.

6. Liu J, Cai M, Chen J, Liao Y, Mai S, Li Y, Huang X, Liu Y, Zhang J, Kung H, Zeng Y, Zhou F, Xie D. $\alpha 4$ contributes to bladder urothelial carcinoma cell invasion and/or metastasis via regulation of E-cadherin and is a predictor of outcome in bladder urothelial carcinoma patients. European Journal of Cancer 2014; 50: 840-51.

7. Wlodarchak N, Xing Y. PP2A as a master regulator of the cell cycle. Crit Rev Biochem Mol Biol. 2016; 51(3): 162-84.

8. Chen $\mathrm{W}$ et al. Identification of specific PP2A complexes involved in human cell transformation. Cancer Cell 2004; 5(2): 127-36.

9. Janssens V, Goris J, Van Hoof C. PP2A: the expected tumour suppressor. Curr Opin Genet Dev 2005; 15: 34-41.

10. Perrotti D, Neviani P. Protein phosphatase 2A: a target for anticancer therapy. Lancet Oncol 2013; 14: 229-38.

11. Marin GH, Rebollo A, Bruzzoni-Giovanelli H, Schinella G, Piazzon I, Duarte A, Errecalde J. T cell leukemia control via Ras-Raf pathway inhibition with peptides. J Med Life 2017; 10(3): 172-5. 